\title{
A NADP-glutamate dehydrogenase mutant of the petit-negative yeast Kluyveromyces lactis uses the glutamine synthetase-glutamate synthase pathway for glutamate biosynthesis
}

\author{
Lourdes Valenzuela, Simón Guzmán-León, Roberto Coria, \\ Jorge Ramírez, Cristina Aranda and Alicia González
}

Author for correspondence: Alicia González. Tei: +52 6225631. Fax: +526225630.

Departamento de

Microbiología, Instituto de Fisiología Celular,

Universidad Nacional

Autónoma de México

Apartado Postal 70-242,

04510 Mexico City, Mexico

\begin{abstract}
The activities of the enzymes involved in ammonium assimilation and glutamate biosynthesis were determined in wild-type and NADP-glutamate dehydrogenase (GDH) null mutant strains of Kluyveromyces lactis. The specific NADP-GDH activity from $K$. lactis was fivefold lower than that found in Saccharomyces cerevisiae. The glutamine synthetase (GS) and glutamate synthase (GOGAT) activities were similar to those reported in S. cerevisiae. The NADP-GDH null mutant was obtained by transforming the uraA strain MD2/1 with a linearized integrative yeast vector harbouring a $390 \mathrm{bp}$ fragment of the NADP-GDH structural gene. This mutant grew as well as the parent strain on ammonium, but showed GS and GOGAT activities higher that those found in the wild-type strain, implying that the GS-GOGAT pathway could play a leading role in glutamate biosynthesis in $K$. lactis. Southern blotting analysis of $K$. lactis chromosomes separated by contour-clamped homogeneous electric field electrophoresis, indicated that the NADP-GDH structural gene is localized on chromosome VI.
\end{abstract}

Keywords: nitrogen metabolism, glutamate biosynthesis

\section{INTRODUCTION}

Two pathways for ammonium assimilation and glutamate biosynthesis have been found in a variety of organisms. In one pathway, NADP-glutamate dehydrogenase (NADPGDH; EC 1.4.1.4) catalyses the reductive amination of 2-oxoglutarate to form glutamate (Holzer \& Schneider, 1957). The existence of an alternative pathway for the net biosynthesis of glutamate, was demonstrated by Tempest et al. (1970). In this pathway, glutamate is aminated to form glutamine by glutamine synthetase (GS; EC 6.3.1.2) and the amide group is then transferred reductively to 2-oxoglutarate by glutamate synthase (GOGAT; EC 1.4.1.13), resulting in the net conversion of ammonium and 2-oxoglutarate to glutamate. The GSGOGAT pathway has been found in several microorganisms (Senior, 1975; Hummelt \& Mora, 1980; Bravo \& Mora, 1988; Marqués et al., 1992) and in higher plants (Miflin et al., 1980).

The presence of two pathways for glutamate biosynthesis

Abbreviations: CHEF, contour-clamped homogeneous electric field; GS, glutamine synthetase; GOGAT, glutamate synthase. has opened a discussion as to the need for two routes for the biosynthesis of the same end-product. In plants, the GS-GOGAT pathway constitutes the main route of ammonium assimilation (Miflin et al., 1980). In Klebsiella aerogenes, the GS-GOGAT pathway functions when the ammonium concentration is low, while the GDH pathway is more active when the cells are cultivated under ammonium excess (Tempest et al., 1970; Meers et al., 1970). In Neurospora crassa, it has been shown that the main function of GOGAT is to recycle some organic nitrogen from glutamine to glutamate, and that both pathways are involved in glutamate synthesis either on excess or limiting ammonium (Lomnitz et al., 1987).

In Eschericbia coli, GDH and GOGAT activities are both present, but their precise functions are not known (Senior, 1975). It had been proposed that in this bacterium, the lack of GOGAT resulted in the incapacity to use a variety of nitrogen sources ( $\mathrm{Ntr}^{-}$phenotype). However, it has now been established that the induction of Ntr enzymes is independent of GOGAT activity and mutants lacking GOGAT activity which are able to induce histidase have been isolated (Castaño et al., 1992). Recently, Helling (1994) proposed that in E. coli, glutamate biosynthesis 
could proceed through NADP-GDH when the cell is energy-limited, while the GS-GOGAT pathway would function when the cell is not energy-limited.

The role of the GDH and GS-GOGAT pathways has been studied in some yeasts. Activities of the two ammonium-assimilation pathways in continuous cultures of Candida albicans and the isolation of Schizosaccharomyces pombe glutamate auxotrophs has indicated that the GSGOGAT pathway is the major pathway for ammonium assimilation in these yeasts (Holmes et al., 1989; Barel \& MacDonald, 1993). Saccharomyces cerevisiae mutants devoid of the biosynthetic NADP-GDH or of GOGAT have also been obtained (Folch et al., 1989; Miller \& Magasanik, 1990; Cogoni et al., 1995). Those strains impaired in NADP-GDH show a twofold slower doubling time as compared to the wild-type strain when these are grown on ammonium as sole nitrogen source (Folch et al., 1989). The lack of GOGAT activity shows no phenotype when this yeast is grown on either high or low ammonium (Folch et al., 1989). Thus, in S. cerevisiae NADP-GDH has been assigned a role in glutamate biosynthesis, while the participation of GOGAT has remained unclear, and this activity would seem dispensable.

We report here catalytic activities of NADP-GDH, GS and GOGAT and the isolation of a NADP-GDH null mutant from Kluyveromyces lactis. The NADP-GDHless mutant strain has no growth-defective phenotype and shows higher GOGAT and GS activities than the wildtype strain, indicating that in this yeast the GS-GOGAT pathway could play a major role in glutamate biosynthesis and ammonium assimilation.

\section{METHODS}

Growth conditions. Strains were routinely grown on minimal medium (MM) containing salts, trace elements, and vitamins following the formula of yeast nitrogen base (Difco). Glucose $(2 \%, w / v)$ was used as the carbon source, and $38 \mathrm{mM}$ $\left(\mathrm{NH}_{4}\right)_{2} \mathrm{SO}_{4}$ was used as the nitrogen source. Amino acids needed to satisfy auxotrophic requirements were added at $0.01 \%(\mathrm{w} / \mathrm{v})$. Cells were incubated at $30^{\circ} \mathrm{C}$ with agitation. Growth was monitored by measuring optical density at $600 \mathrm{~nm}$ (Hewlett Packard model 8452A spectrophotometer).

Determination of NADP-GDH, GS and GOGAT activities. Soluble extracts for enzyme assays were prepared by grinding whole cells suspended in $0 \cdot 1 \mathrm{M}$ potassium phosphate, $\mathrm{pH} 7 \cdot 5$, $1 \mathrm{mM}$ EDTA, with glass beads and a Vortex mixer (six cycles of $1 \mathrm{~min}$ ). NADP-GDH, GS and GOGAT were assayed by the methods of Doherty (1970), Ferguson \& Sims (1974) and Roon et al. (1974), respectively.

Oligonucleotide design and PCR amplification of $K$. lactis genomic DNA. Two pairs of degenerated deoxyoligonucleotides were designed based on the amino acid sequence of $S$. cerevisiae GDH (Nagasu \& Hall, 1985; Moye et al., 1985) and K. lactis codon usage (Lloyd \& Sharp, 1993).

The deoxyoligonucleotide $\mathrm{F} 1, \quad \mathrm{TT}(\mathrm{G}+\mathrm{A}) \quad \mathrm{GA}(\mathrm{A}+\mathrm{G})$ $\mathrm{GA}(\mathrm{C}+\mathrm{T}) \mathrm{TC}(\mathrm{T}+\mathrm{C}) \mathrm{AC}(\mathrm{T}+\mathrm{C}) \mathrm{CT}(\mathrm{T}+\mathrm{C}) \mathrm{TT}(\mathrm{T}+\mathrm{C}) \mathrm{GA}$ was designed from the sequence LEDSTLFE located at position 17-24 in the GDH sequence. The deoxyoligonucleotide R1, $\mathrm{TT}(\mathrm{C}+\mathrm{T}) \quad \mathrm{AA}(\mathrm{G}+\mathrm{A}) \quad \mathrm{GC}(\mathrm{G}+\mathrm{A}) \quad \mathrm{GC}(\mathrm{G}+\mathrm{A}) \quad \mathrm{TA}(\mathrm{T}+\mathrm{C})$ $T G(A+G) G C(A+G) A C(G+A) T T$ was designed from the sequence NVAQYAALK located at position 227-235. The deoxyoligonucleotide $\mathrm{F} 2$, TT(G+A) $\mathrm{GA}(\mathrm{G}+\mathrm{A}) \mathrm{CA}(\mathrm{A}+\mathrm{G})$ $\mathrm{AT}(\mathrm{C}+\mathrm{T}) \mathrm{GT}(\mathrm{C}+\mathrm{T}) \mathrm{AA}(\mathrm{C}+\mathrm{T}) \mathrm{GA}(\mathrm{G}+\mathrm{A}) \mathrm{TA}(\mathrm{C}+\mathrm{T}) \mathrm{TC}$ was designed from the sequence LEQIVNEYS located at position 277-285. The deoxyoligonucleotide R2, CAT $(\mathrm{A}+\mathrm{G}) \mathrm{AT} \quad(\mathrm{T}+\mathrm{C}) \mathrm{CT} \quad(\mathrm{T}+\mathrm{C}) \mathrm{TT} \quad(\mathrm{C}+\mathrm{T}) \mathrm{AA} \quad(\mathrm{C}+\mathrm{T}) \mathrm{TC}$ $(\mathrm{T}+\mathrm{C}) \mathrm{TG}(\mathrm{G}+\mathrm{A}) \mathrm{TC}$ was designed from the sequence DQELKRIM located at position 400-407.

Total DNA from $K$. lactis strain WM37 (MAT a, his 3 ) was used as template for amplification by PCR. It was carried out in a Coy TempCycler II with the following program: one denature cycle for $10 \mathrm{~min}$ at $94^{\circ} \mathrm{C}$, followed by 50 cycles of $30 \mathrm{~s}$ denaturation at $94^{\circ} \mathrm{C}, 45 \mathrm{~s}$ annealing at $45^{\circ} \mathrm{C}$ and $2 \mathrm{~min}$ extension at $72{ }^{\circ} \mathrm{C}$, with a final 10 min extension at $72{ }^{\circ} \mathrm{C}$. A 390 bp PCR product obtained from primers $\mathrm{F} 2-\mathrm{R} 2$ was gel-purified and ligated into the PCRII vector (Invitrogen). This subclone was sequenced by primer extension using the sequenase V.2 kit (USB).

Chromosome separation. Chromosomes from strain WM37 were separated by contour-clamped homogeneous electric field (CHEF) electrophoresis as described by Miranda et al. (1995).

Southern blot analysis. The agarose gels containing BglII restricted DNA from the wild-type and NADP-GDH null mutant and that containing separated chromosomes were exposed to short wavelength UV for 5 min to break the DNA, then denatured, neutralized and transferred to nylon membranes as described in Sambrook et al. (1989). DNA blots were probed with the 390 bp PCR fragment labelled with $\left[\alpha^{32}\right.$ P]dCTP.

\section{RESULTS AND DISCUSSION}

\section{Determination of the enzymes involved in glutamate biosynthesis}

Glutamate biosynthesis can be achieved through the action of the NADP-GDH or through the concerted action of GS and GOGAT. To determine whether these two pathways were present in $K$. lactis the activity of these enzymes was measured (Fig. 1). In extracts of the wildtype strain grown on ammonium as sole nitrogen source, GS activity increased slightly in the stationary growth phase, while GOGAT activity was highest during the exponential growth phase. The specific activities of these two enzymes were similar to those found in $S$. cerevisiae (González et al., 1985a; Folch et al., 1989). NADP-GDH showed the highest activity during exponential phase of $K$. lactis, although the specific activity was fivefold lower than that reported for S. cerevisiae (González et al., 1985b). It is worth noting that even though $K$. lactis showed a considerably lower NADP-GDH activity than S. cerevisiae, both strains grew on ammonium with the same doubling time $(2 \mathrm{~h})$. This result implies that in $K$. lactis the growth rate on ammonium is not limited by the capacity to assimilate ammonium through the NADP-GDH, but that the normal assimilation of ammonia in $K$. lactis could depend on the GS-GOGAT pathway. To resolve this question, $K$. lactis mutants devoid of NADP-GDH activity were isolated.

\section{Isolation and characterization of a NADP-GDH null mutant}

Based on the observation that there is a high degree of amino acid sequence similarity between various proteins from S. cerevisiae and K. lactis (Hendriks et al., 1992) and 

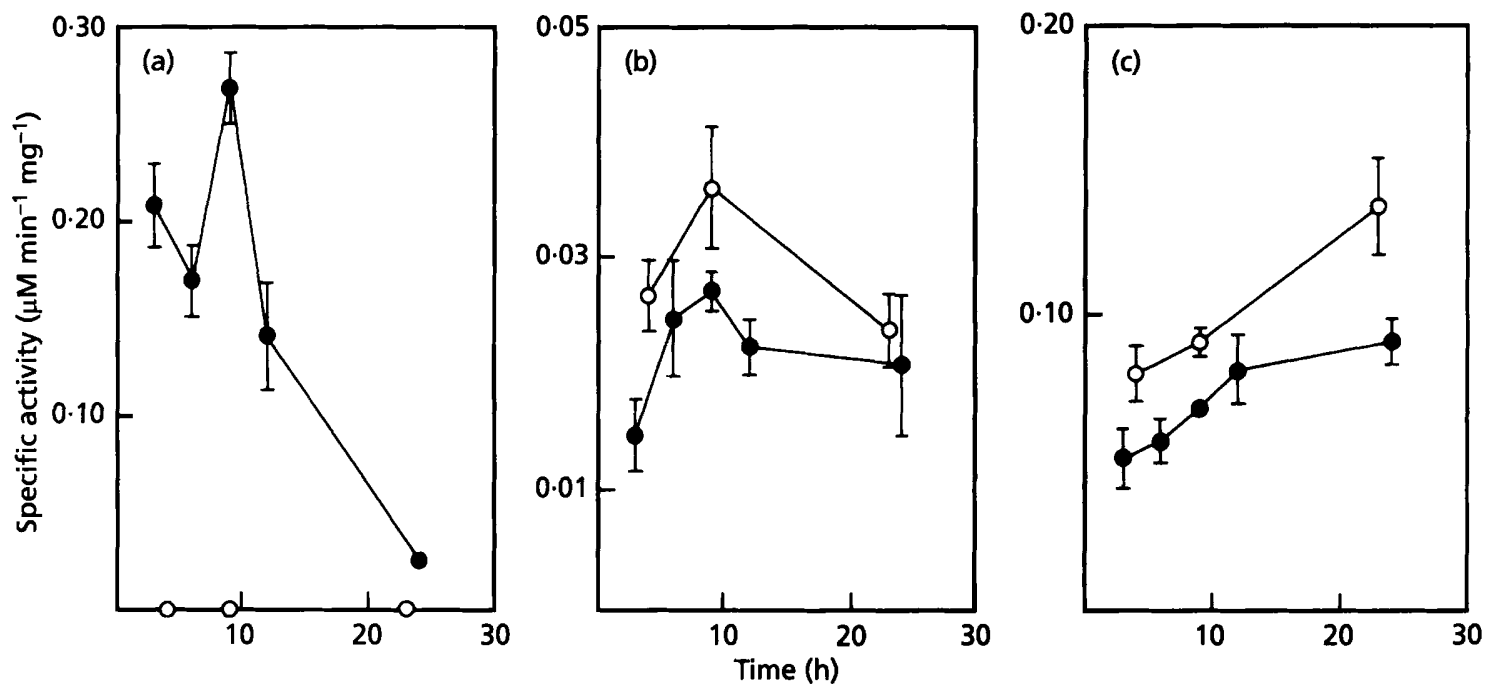

Fig. 1. Specific activities of (a) NADP-GDH, (b) GOGAT and (c) GS in the wild-type strain (O) and in the mutant strain JR1 (O). Extracts were obtained from cells grown on $\left(\mathrm{NH}_{4}\right)_{2} \mathrm{SO}_{4}$ as sole nitrogen source. Bars represent SD of four experiments.

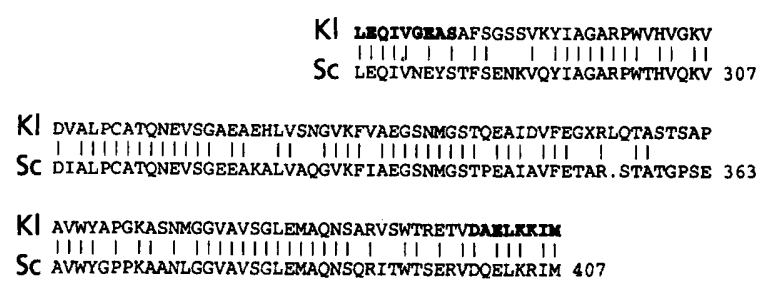

Fig. 2. Amino acid sequence alignment of $K$. lactis (KI) PCRamplified NADP-GDH fragment with NADP-GDH protein from $S$. cerevisiae (Sc).

the fact that codon usage is very similar in these two yeasts (Lloyd \& Sharp, 1993), two pairs of deoxyoligonucleotides were designed as amplification primers to be used on total DNA from K. lactis strain WM37. According to the $S$. cerevisiae NADP-GDH sequence (Moye et al., 1985; Nagasu \& Hall, 1985), primers F1/R1 (see Methods), were expected to amplify a $654 \mathrm{bp}$ fragment; primers F2/R2 were expected to amplify a $390 \mathrm{bp}$ fragment, while the combined primers $\mathrm{F} 1-\mathrm{R} 2$ were expected to amplify a $1170 \mathrm{bp}$ fragment. Only the F2/R2 couple amplified the expected product. This $390 \mathrm{bp}$ fragment was subcloned into the pCRII vector and sequenced in its entirety. The deduced amino acid sequence showed a high degree of similarity $(75 \%$ similarity, $67 \%$ identity) with the corresponding region of the NADP-GDH protein from S. cerevisiae (Fig. 2).

To obtain a $K$. lactis NADP-GDH null mutant, we constructed plasmid pJR1 by cloning the 390 bp PCR fragment into YIp352 integrative vector, which harbours the $U R A 3$ yeast marker. The resulting plasmid, pJR1, was linearized by digesting at the single ClaI site located at position 214 of the PCR fragment. Strain MD2/1 $(M A T \alpha \operatorname{lys} A \arg A \operatorname{ura} A)$ was transformed to uracil

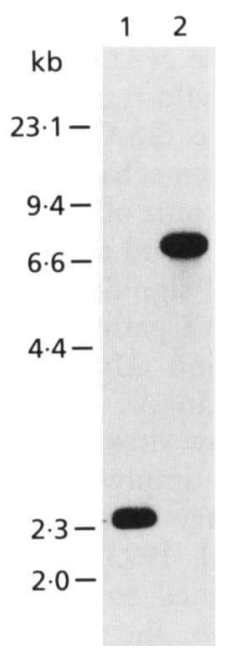

Fig. 3. Southern analysis of DNA digested with Bg/ll obtained from $K$. lactis. Lanes: 1, wild-type strain; 2, mutant strain JR1. The blot was probed with a 390 bp PCR fragment internal to the NADP-GDH gene.

prototrophy. Chromosomal DNA was isolated from 10 transformants and digested with HindIII. Southern analysis was carried out with the $390 \mathrm{bp}$ PCR fragment as the probe. The pattern of transformants clearly indicated the insertion of the construct in the wild-type genomic sequence of NADP-GDH (Fig. 3). The null mutants obtained were completely devoid of NADP-GDH activity (Fig. 1a).

The doubling time of the wild-type and of the NADPGDH mutant strains when both were grown on ammonium as sole nitrogen source, was $120 \mathrm{~min}$ (data not shown). These results imply, that in $K$. lactis, NADPGDH could be a non-essential enzyme under these growth 
(a)

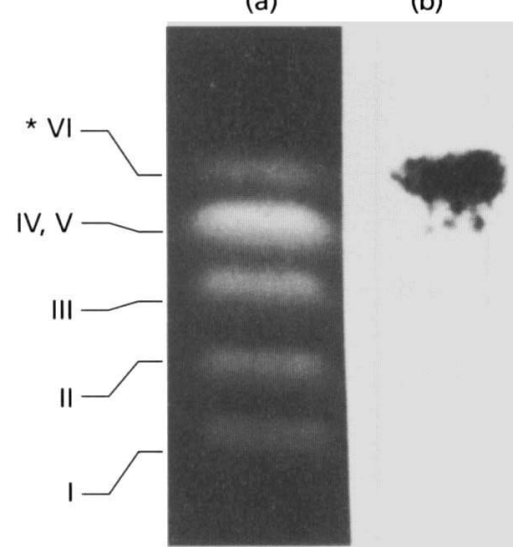

Fig. 4. (a) Chromosome pattern of the $K$. lactis strain WM37 separated by CHEF electrophoresis and stained with ethidium bromide. (b) Autoradiography of a blot showing hybridization of chromosome VI ${ }^{*}{ }^{*}$ to the ${ }^{32}$ P-labelled 390 bp GDH PCR fragment.

conditions. GOGAT and GS specific activities were 1.5to 2-fold higher in the NADP-GDH mutant strain than those found in the wild-type strain (Fig. 1b, c). These results imply that the GS-GOGAT pathway could be either a compensatory mechanism for the lack of NADPGDH or the primary route of ammonium assimilation and glutamate biosynthesis and that the NADP-GDH could have only a collateral significance. In $C$. albicans and $S_{C}$. pombe, the GS-GOGAT pathway plays an essential role in ammonium assimilation (Holmes et al., 1989; Barel \& MacDonald, 1993). In Sc. pombe, mutants devoid of GOGAT activity grow slowly and increase fivefold their NADP-GDH levels implying that the GS-GOGAT pathway is the primary route of glutamate biosynthesis (Barel \& MacDonald, 1993). It can be concluded that while the yeasts studied to date have both routes for glutamate biosynthesis, the primary route is not always the same.

\section{Chromosomal localization of the NADP-GDH structural gene}

Using the CHEF conditions described in Methods, six bands were separated that corresponded to chromosomes from strain WM37 (MATa, bis3) (Fig. 4, a), that match with those reported from the strain CBS 2360 (Sor \& Fukuhara, 1989). These chromosomes, numbered I to VI, were immobilized on a nylon membrane. The $K$. lactis GDH1 (NADP-GDH) gene was localized on chromosome VI using the PCR-amplified fragment as probe (Fig. 4, b). The K. lactis GDH1 gene encoding the NADPGDH should be added to the growing list of genes mapped in this organism.

\section{ACKNOWLEDGEMENTS}

The authors are grateful to Hiram Olivera and Lourdes Miranda for their critical review of the manuscript. This work was supported in part by the Dirección General de Asuntos del Personal Académico, Universidad Nacional Autónoma de México (IN201894).

\section{REFERENCES}

Barel, I. \& MacDonald, D. W. (1993). Enzyme defects in glutamaterequiring strains of Schizosaccharomyces pombe. FEMS Microbiol Lett 113, 267-272.

Bravo, A. \& Mora, J. (1988). Ammonium assimilation in Rhizobium phaseoli by the glutamine synthetase-glutamate synthase pathway. $J$ Bacteriol 170, 980-984.

Castaño, I., Flores, N., Valle, F., Covarrubias, A. A. \& Bolivar, F. (1992). gltF, a member of the gltBDF operon of Escherichia coli, is involved in nitrogen-regulated gene expression. Mol Microbiol 6, 2733-2741.

Cogoni, C., Valenzuela, L., González-Halphen, D., Olivera, H., Macino, G., Ballario, P. \& González, A. (1995). Saccbaromyces cerevisiae has a single glutamate synthase gene coding for a plant-like high-molecular-weight polypeptide. J Bacteriol 177, 792-798.

Doherty, D. (1970). L-Glutamate dehydrogenases (yeast). Methods Enzymol 17, 850-856.

Ferguson, A. R. \& Sims, A. P. (1974). The regulation of glutamine metabolism in Candida utilis: the role of glutamine in the control of glutamine synthetase. $J$ Gen Microbiol 80, 159-171.

Folch, J. L., Antaramián, A., Rodríguez, L., Bravo, A., Brunner, A. \& González, A. (1989). Isolation and characterization of a Saccharomyces cerevisiae mutant with impaired glutamate synthase activity. J Bacteriol 171, 6776-6781.

González, A., Dávila, G. \& Calva, E. (1985a). Cloning of a DNA sequence that complements glutamine auxotrophy in Saccharomyces cerevisiae. Gene 36, 123-129.

González, A., Rodríguez, L., Olivera, H. \& Soberón, M. (1985b). NADP-dependent glutamate dehydrogenase activity is impaired in mutants of Saccharomyces cerevisiae that lack aconitase. J Gen Microbiol 131, 2565-2571.

Helling, R. B. (1994). Why does Escherichia coli have two primary pathways for synthesis of glutamate? J Bacteriol 176, 4664-4668.

Hendriks, L., Goris, A., Van de Peer, Y., Neefs, Y. M., Vancanneyt, M., Kersters, K., Berny, J. F., Hennebert, G. L. \& De Waechter, R. (1992). Phylogenetic relationships among ascomycetes and ascomycetes-like yeasts as deduced from small ribosomal subunit RNA sequences. Syst Appl Microbiol 15, 98-104.

Holmes, A. R., Collings, A., Farnden, K. J. F. \& Sheperd, M. G. (1989). Ammonium assimilation by Candida albicans and other yeasts: evidence for activity of glutamate synthase. $J$ Gen Microbiol 135, 1423-1430.

Holzer, H. \& Schneider, S. (1957). Anreicherung und Trennung einer DPN-spezifischen und einer TPN-spezifischen Glutaminosaure Dehydrogenase aus Hefe. Biochem Z 329, 361-367.

Hummelt, G. \& Mora, J. (1980). Regulation and function of glutamate synthase in Neurospora crassa. Biochem Biophys Res Commun 96, 1688-1694.

Lloyd, A. T. \& Sharp, P. M. (1993). Synonymous codon usage in Kluyveromyces lactis. Yeast 9, 1219-1228.

Lomnitz, A., Calderón, J., Hernández, G. \& Mora, J. (1987). Functional analysis of ammonium assimilation enzymes in Neurospora crassa. J Gen Microbiol 133, 2333-2340.

Marqués, S., Florencio, F. J. \& Candau, P. (1992). Purification and characterization of the ferredoxin-glutamate synthase from the unicellular cyanobacterium Synecbococcus sp. PCC 6301. Eur J Biochem 206, 69-77. 
Meers, J. L., Tempest, D.W. \& Brown, C. M. (1970). Glutamine(amide): 2-oxoglutarate amino transferase oxidoreductase (NADP), an enzyme involved in the synthesis of glutamate by some bacteria. J Gen Microbiol 64, 187-194.

Miflin, B. J., Lea, P. J. \& Wallsgrove, R. M. (1980). The role of glutamine in ammonium assimilation and reassimilation in plants. In Glutamine: Metabolism, Enzymology and Regulation, pp. 213-234. Edited by J. Mora \& R. Palacios. NY: Academic Press.

Miller, S. M. \& Magasanik, B. (1990). Role of NAD-linked glutamate dehydrogenase in nitrogen metabolism in Saccharomyces cerevisiae. J Bacteriol 172, 4927-4935.

Miranda, M., Ramírez, J., Guevara, S., Ongay-Larios, L., Peña, A. \& Coria, R. (1995). Nucleotide sequence and chromosomal localization of the gene encoding the old yellow enzyme from Kluyveromyces lactis. Yeast 11, 459-465.

Moye, W. S., Amuro, N., Rao, J. K. M. \& Zalkin, H. (1985). Nucleotide sequence of yeast GDH1 encoding nicotinamide adenine dinucleotide phosphate-dependent glutamate dehydrogenase. J Biol Chem 260, 8502-8508.

Nagasu, T. \& Hall, B. (1985). Nucleotide sequence of the GDH gene coding for the NADP-specific glutamate dehydrogenase of Saccharomyces cerevisiae. Gene 37, 247-253.

Roon, R. J., Even, H. L. \& Larimore, F. (1974). Glutamate synthase: properties of the reduced nicotinamide adenine dinucleotidedependent enzyme from Saccharomyces cerevisiae. I Bacteriol 118, 89-95.

Sambrook, J., Fritsch, E. F. \& Maniatis, T. (1989). Molecular Cloning: a Laboratory Manual, 2nd edn. Cold Spring Harbor, NY: Cold Spring Harbor Laboratory.

Senior, P. J. (1975). Regulation of nitrogen metabolism in Escherichia coli and Klebsiella aerogenes: studies with the continuousculture technique. $J$ Bacteriol 123, 407-418.

Sor, F. \& Fukuhara, H. (1989). Analysis of chromosomal DNA patterns of the genus Kluyveromyces. Yeast 5, 1-10.

Tempest, D. W., Meers, J. L. \& Brown, C. M. (1970). Synthesis of glutamate in Aerobacter aerogenes by a hitherto unknown route. Biocbem J 117, 405-407.

Received 16 March 1995; revised 22 June 1995; accepted 27 June 1995. 